A Case of Lethal Genes in the Horse

THE only case of a lethal gene in the horse described hitherto was by Yamane $^{1}$ in Japan; it produced a blind large intestine, which caused colic and death among the new-born foals sired by one Percheron stallion. The following case, which I have observed in the well-known Polish stud of halfbreed horses at Skrzydlów, p-Aurelów, near Czestochowa, is therefore of interest, because its explanation is analogous to that of Yamane's case mentioned above, although perhaps more complicated.

The Anglo-Arab stallion Menzin (Polish St. B. V. I., p. 204) covered in 1933 seventeen mares; in 1934 , nine foals sired by Menzin were born, of which six were quite normal and three showed a peculiar lameness of one or both forelegs. The new-born foals could not stand at all and, of course, it was necessary to destroy them. In the following year (1935) thirteen foals were sired by Menzin : of these nine were normal and four were cripples. In neighbouring stud-farms, where Menzin also covered some mares, four foals sired by him were born; three were normal and one cripple.

It should be stated that, before 1934, when the mares at Skrzydlów were served by other stallions, they never produced cripples.

The legs of the crippled foals were examined and no signs of rickets or any other bone disease have been found. The lameness consisted in a marked bending of the leg in the region of the phalanges, with an undeveloped hoof. Somewhat analogous with this is the case of inherited crooked fingers in new-born children described by Mohr ${ }^{2}$.

A detailed report in a genetical journal will follow.

Zootechnical Laboratory, R. T. Prawochenski.

Jagellonian University, Cracow.

1 "Biological Essays on the Horse", 1929, p. 39. Berlin.

2 "Utber Lethalfaktoren", Z. ind. Abst. u. Ver. Lehre, 1926.

\section{Mutations arising at the Bar Locus in Drosophila melanogaster}

THE Bar mutation has been investigated by Sturtevant (1925-26), Dobzhansky (1928) and others. However, so far two very important lines of inquiry have remained obscure in the Bar problem : (1) What is the cause of the occurrence of Bar mutation de novo ; and (2) what is the nature of Bar mutations caused by irradiation with X-rays ?

Hanson (1928) has shown that Bar mutations arise in males exposed to X-rays. By exposing males to $\mathrm{X}$-rays we obtained a great number of Bar mutations (1:382) ; for example, complete reverse mutations, feebler and stronger allelomorphs of Bar, some of which were lethal, others sterile. A cytogenetic study of the salivary nuclei has shown that out of thirty-four cases studied, in seventeen the occurrence of the Bar mutation was connected with an aberration. In ten of these cases one break occurred exactly at the Bar locus (Fig. 1); in six cases, some distance from Bar; and in one case there was a deficiency which included the Bar locus. It is noteworthy that among the complete reverse mutations studied, only one aberration among ten was connected with a break at a distance from Bar; whereas in the seven cases of incomplete reverse mutations (infra-Bar and ultra-Bar types), five breaks occurred some distance from Bar.

In the majority of cases the complete reverse Bar mutations are not connected with a loss of this locus. They are not manifested phenotypically, however, when they are heterozygous for Bar, appearing as homozygous Bar. On the other hand, the reverse mutations retained completely the mutational potentialities of Bar. When reverse mutations were exposed to X-rays a great number of Bar mutations were obtained (1:928) which were all connected with chromosome aberrations. The eight cases studied cytologically were all connected with a break of the $X$-chromosome at Bar.

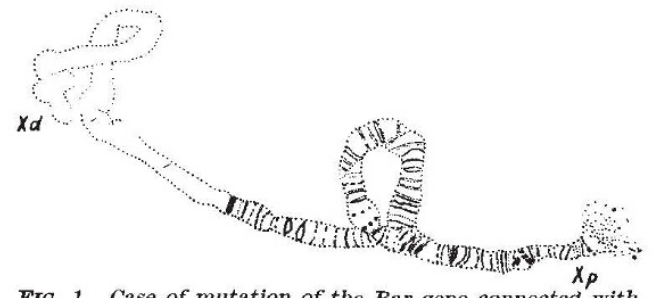

FIG. 1. Case of mutation of the Bar gene connected with aberration at the Bar locus (inversion).

The X-irradiation of double Bar showed unexpectedly a lower frequency of Bar mutations and a disproportionately frequent simultaneous mutation of both Bar genes, producing an allelomorph without phenotypic manifestation (double reverse Bar but not deficiency as Demerec thought). Finally, it was shown that half of these mutations occur in connexion with chromosome aberrations-of fourteen cases six were without aberrations and eight were connected with breaks at the Bar locus.

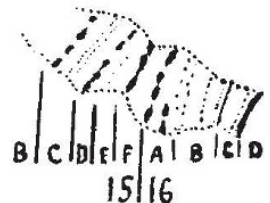

15116 $X$-chromosome from the nucleus of a saliheterozygous fly (double-Bar $\hat{X}$ Normal). The insertion of double Bar is detectable in region $16 \mathrm{~A}$.
FIG. 2. A region of the

Taking into account Sturtevant's investigations and Wright's considerations, we investigated Bar cytologically. In the females heterozygous for Bar, an insignificant non-coincidence of chromosomes at the Bar locus was found. A study of the hetero. zygotes for double Bar (E. N. Volotov) has shown the presence of insertions at the Bar locus (Fig. 2). Consequently, all the known occurrences of Bar mutations de novo are connected with disturbances of the chromosomes at the Bar locus (the case of Tice which was studied eytologically by us, baroid of Dobzhansky, the two Bar mutations of Muller, and one Bar mutation discovered by Dubinin and Goldat, unpublished).

The mutational changes of the Bar gene induced by $\mathrm{X}$-rays are in many instances caused by the effect of the position of this gene on its manifestation.

The Bar mutations occurring de novo are also caused by the effect of the position of the genes at the Bar locus (see also Dobzhansky, 1932).

A study of the occurrence of Bar mutations under the action of $\mathrm{X}$-rays shows that the breaks at the Bar locus occur more frequently than Bar mutations de novo (if the greater frequency of breaks in the Bar chromosome are not caused by the presence of the Bar gene itself). Hence, for the occurrence of Bar de novo not only a break of the chromosome at the Bar locus is necessary but also an attachment of some other specific material as well.

A comparison of the mutational process in Bar and double Bar leads us to suspect that the position effect changes the action of the gene as well as its mutational potentialities.

N. P. DubrNin.

E. N. Volotov.

Institute of Experimental Biology, Moscow. 